\title{
Hymenoscyphus linearis sp. nov: another close relative of the ash dieback pathogen $H$. fraxineus
}

\author{
A. Gross • T. Hosoya ・ Y.-J. Zhao • H.-O. Baral
}

Received: 2 October 2014 /Revised: 24 January 2015 / Accepted: 10 February 2015 /Published online: 29 March 2015

(C) German Mycological Society and Springer-Verlag Berlin Heidelberg 2015

\begin{abstract}
Hymenoscyphus is a large fungal genus containing a single known severe pathogen (H. fraxineus) causing ash dieback in Europe. Here, the closely related species Hymenoscyphus linears sp. nov. is described from Japan. Apothecia of this fungus emerge from linear, black pseudosclerotia on rachises and petioles of Fraxinus platypoda. In culture, the species forms a Chalara anamorph similar to that of $H$. fraxineus. In addition, a Sporotrichum-like synanamorph is produced. Spores of neither anamorphic forms germinate on malt extract agar and might act solely as spermatia. After prolonged incubation, ascocarps are produced directly on agar plates, indicating the ability to self-fertilize. Phylogenetic investigations using four sequence markers placed the species alongside $H$. albidus, $H$. albidoides, and $H$. fraxineus. Eight fungal strains, co-occurring with $H$. linearis on petioles of $F$. platypoda, were isolated and identified by sequencing the ITS region of the rDNA. Seven different species were revealed, showing that there is no other dominant fungus on petioles of F. platypoda. Pathogenicity tests on Fraxinus excelsior and its native host $F$. platypoda revealed that the fungus
\end{abstract}

Electronic supplementary material The online version of this article (doi:10.1007/s11557-015-1041-3) contains supplementary material, which is available to authorized users.

A. Gross $(\bowtie)$

ETH Zurich, Department of Environmental Systems Sciences, Forest Pathology and Dendrology, Institute of Integrative Biology (IBZ), ETH Zurich, 8092 Zurich, Switzerland

e-mail: andrin.gross@usys.ethz.ch

T. Hosoya $\cdot$ Y.-J. Zhao

Department of Botany, National Museum of Nature and Science,

4-1-1, Amakubo, Tsukuba, Ibaraki, Japan

H.-O. Baral

Blaihofstr. 42, Tübingen 72074, Germany is avirulent. The close phylogenetic relationship with $H$. fraxineus and the avirulence of $H$. linearis offer an opportunity to study the evolution of pathogenicity of $H$. fraxineus in further detail in the future.

Keywords Chalara fraxinea $\cdot$ Hymenoscyphus pseudoalbidus · Molecular phylogeny · Fraxinus platypoda . Hymenoscyphus albidus · Virulence assay

\section{Introduction}

The fungal genus Hymenoscyphus (class Leotiomycetes, order Helotiales) has received increasing attention since $H$. albidus (Roberge ex Gillet) W. Phillips s. 1. was linked to the novel tree disease European ash dieback (Kowalski and Holdenrieder 2009). Later, Hymenoscyphus pseudoalbidus Queloz, Grünig, Berndt, T. Kowalski, T.N. Sieber \& Holdenr. was newly described as a cryptic sister species of $H$. albidus and proven to be the causal agent of the disease (Queloz et al. 2011). Subsequently, H. pseudoalbidus, now known as H. fraxineus (Baral et al. 2014), was found to be native to East Asia, where it was confirmed in Japan (Zhao et al. 2012; Hosoya et al. 1993), Korea (J. Han, personal communication), east Russia (Marčiulynienè et al. 2013), and China (Zheng and Zhuang 2013). A population genetic comparison of Asian and European $H$. fraxineus demonstrated a strong founder effect in European populations and provided evidence of the species' introduction to Europe via a very small number of individuals (Gross et al. 2014b). Whereas H. albidus is only known from petioles of Fraxinus excelsior and Fraxinus angustifolia (Oleaceae, Lamiales), H. fraxineus has been reported from $F$. excelsior and F. angustifolia (in Europe), and F. mandshurica (east Russia, China, Japan, Korea) and F. rhynchophylla (Korea). Recently, H. albidoides, which is closely related to $H$. albidus, was detected on rotten Picrasma 
quassioides (Simaroubaceae, Sapindales) leaves in China (Zheng and Zhuang 2013). All of these Hymenoscyphus species produce small, whitish apothecia on pseudosclerotial petioles and rachises (hereafter abbreviated as petioles) in the litter of the previous year's leaf-fall.

The ongoing ash dieback epidemic will have a serious impact on the European ash population and associated organisms (Pautasso et al. 2013). In order to preserve the few tolerant ash genotypes (see McKinney et al. 2014) and to avoid virulence evolution of the pathogen, it is essential to preserve the currently low genetic diversity of $H$. fraxineus in Europe (Gross et al. 2014b). Besides the introduction of new genotypes of $H$. fraxineus, closely related species could hybridize with $H$. fraxineus and lead to instances of undesirable evolution (Olson and Stenlid 2002; Brasier and Kirk 2010; Brasier 2000). Therefore, knowledge of the Hymenoscyphus species, which are closely related to $H$. fraxineus, is crucial.

In this paper, we describe a new Hymenoscyphus species that has been found on petioles of Fraxinus platypoda in Japan.

\section{Material methods}

Sampling, isolation, and production of apothecia

Petioles of F. platypoda (synonym: Fraxinus spaethiana) were collected from the leaf litter in the Chichibu forest, University of Tokyo, on 21 May 2012. In the laboratory, the loose epidermal tissues of the petioles were removed by hand, and the surface underneath was sterilized with $100 \%$ ethanol using an aerosol can. Once the alcohol had completely evaporated from the petioles, which had been laid out on a paper tissue, the line-shaped pseudosclerotial structures (sensu Gross and Holdenrieder 2013, see Fig. 1a,b) were tangentially cut with a sterile scalpel, and small tissue samples from the inside of the pseudosclerotium were transferred to ash leaf malt extract agar (AMA) plates supplied with the antibiotic oxytetracycline (Gross et al. 2014b). The plates were incubated at $20{ }^{\circ} \mathrm{C}$ in the dark. In addition, using the same technique, of ten different $F$. platypoda petioles, five tissue samples in close proximity to each other, were taken adjacent to the pseudosclerotia to identify co-occurring fungi on these petioles. The remaining petioles were incubated in a moist chamber at near-UV light, as described in Gross and Holdenrieder (2013), until apothecia developed. Petioles containing mature apothecia were selected as holotype and deposited, together with a living single spore isolate, in the Biological Resource Center, National Institute of Technology and Evaluation (NITE-BRC) in Tokyo, Japan (holotype-ID: TNS-F-52072; type-culture-ID: NBRC 110603). An isotype was deposited at the Swiss mycological herbarium of the ETH Zurich, Switzerland (isotype-ID: ZT Myc 57167) and a duplicate
Fig. 1 Morphology of $H$. linearis (TNS-F-48925): a Petioles of $F$. platypoda showing linear pseudosclerotia, which are frequently connected to leaflet scars (arrows). b Apothecia emerging from pseudosclerotia after incubation in a moist chamber at near-UV light. Note that depending on the state of degradation, the pseudosclerotium might be hidden beneath peripheral tissues. c Apothecia on petioles of $F$. platypoda collected in nature (TNS-F48366). d White apothecia arising from an agar piece used to inoculate sterilized ash petioles on an AMA plate incubated at $20^{\circ} \mathrm{C}$ in the dark. e Yellow apothecia arising from an agar piece used to inoculate sterile ash petioles on an AMA plate, incubated at near-UV light. f Vertical section of an apothecium arising from the pseudosclerotium (in LA). $\mathbf{g}$ Crystals observed in the basal part of the stipe. $\mathbf{h}$ Cross-section through petiole showing black zone-lines of the pseudosclerotial plate forming around sclerenchyma. i Cross-section through fresh petiole as reference to interpret $\mathbf{h}$. Note that epidermis, collenchyma, and cortical chlorenchyma cell layers (arrow) are missing in $\mathbf{h}$. $\mathbf{j}$ Vertical section through fresh apothecium showing brownpigmented cells of the outermost cell layer of the ectal excipulum. $\mathbf{k}$ Ectal and medullary excipulum of rehydrated apothecium (in LA). 1 Ascus of a rehydrated apothecium in MLZ. m Ascus of a fresh apothecium in $\mathrm{H}_{2} \mathrm{O}$. n Ascal apex of a dried apothecium with pores stained blue (arrow) in Lugol's solution without $\mathrm{KOH}$ pretreatment. o Ascal base in Congo red showing no croziers. p Paraphysis mounted in CB/LA. q Paraphyses of dried apothecium mounted in MLZ without $\mathrm{KOH}$ pretreatment. $\mathbf{r}$ Living ascospores mounted in $\mathrm{H}_{2} \mathrm{O}$. $\mathbf{s}$ Germinated ascospores showing germ tubes (in LA). Note that ascospores turn brown and one septate prior to germination. $t$ Surface-view of the pseudosclerotial plate on petioles showing tightly-packed and stronglymelanized hyphae

of the type culture is maintained in the CBS culture collection in Utrecht, Netherlands (CBS-ID: CBS 138754). Additionally, two paratypes (TNS-F-48366, TNS-F-48369) and paratype cultures (NBRC 110921 and 110922, respectively) were deposited at the NITE-BRC in Tokyo, Japan.

\section{Morphological characterization}

Microscopic examinations were carried out using an Olympus BX51-equipped Nomarski phase interference microscope with a digital photograph system (Nikon DS-5 M). Line drawings were prepared with the aid of an Olympus U-DA drawing tube.

To obtain freezing microtome sections, dried materials were rinsed in a drop of $70 \%$ ethanol, re-hydrated in water, embedded in mucilage (Tissue Tek II; Miles Laboratories, Inc., Naperville, IL, USA), frozen on Electrofreeze (MC-802A, Yamato Koki, Tokyo, Japan) and sliced at $15-25 \mu \mathrm{m}$ on a microtome (FX-8001, Yamato Koki, Tokyo, Japan). The sliced materials were mounted in Melzer's reagent (MLZ; $0.5 \mathrm{~g}$ of iodine $\left(\mathrm{I}_{2}\right), 1.5 \mathrm{~g}$ of potassium iodide $(\mathrm{KI}), 20 \mathrm{~g}$ of chloral hydrate $\left(\mathrm{C}_{2} \mathrm{H}_{3} \mathrm{Cl}_{3} \mathrm{O}_{2}\right), 20 \mathrm{ml}$ of distilled water), Lugol's solution (IKI; $1 \mathrm{~g}$ of $\mathrm{I}_{2}, 1 \mathrm{~g}$ of $\mathrm{KI}$ in $100 \mathrm{ml}$ water), cotton blue dissolved in lactic acid $(\mathrm{CB} / \mathrm{LA})$, water or LA for observation. The color codes followed the Pantone color code, adopting the CYMK system, referring to a Pantone color bridge (Anonymous 2005). 

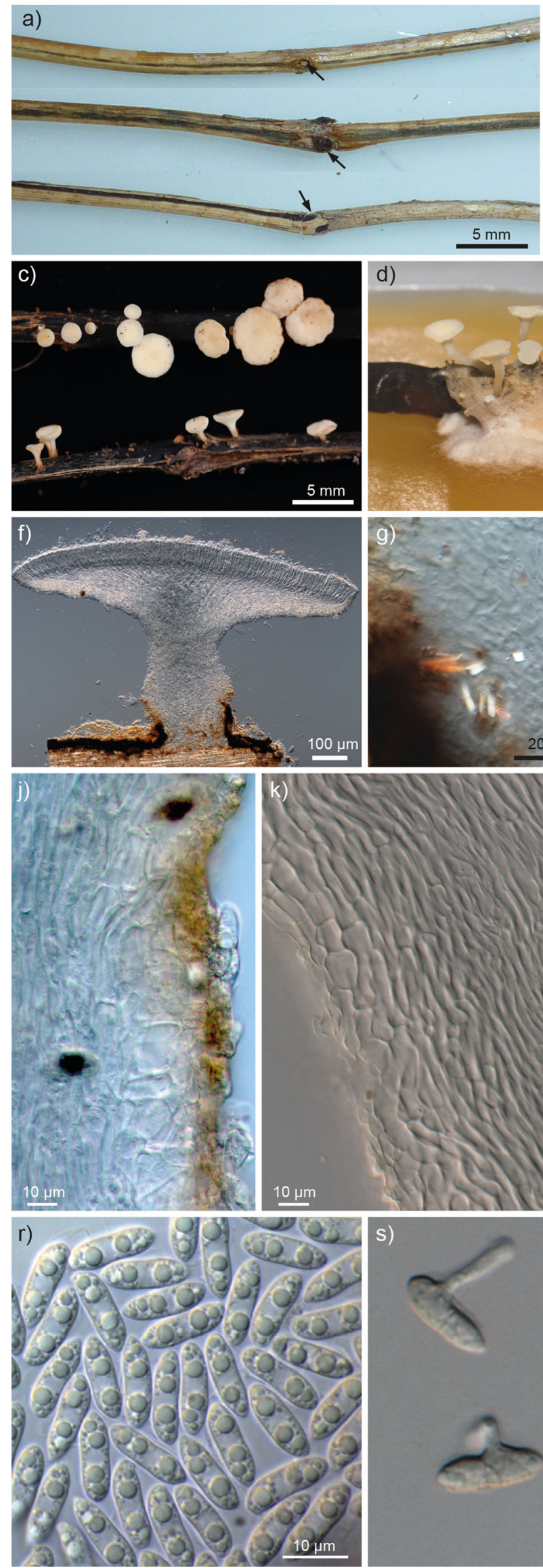

d)
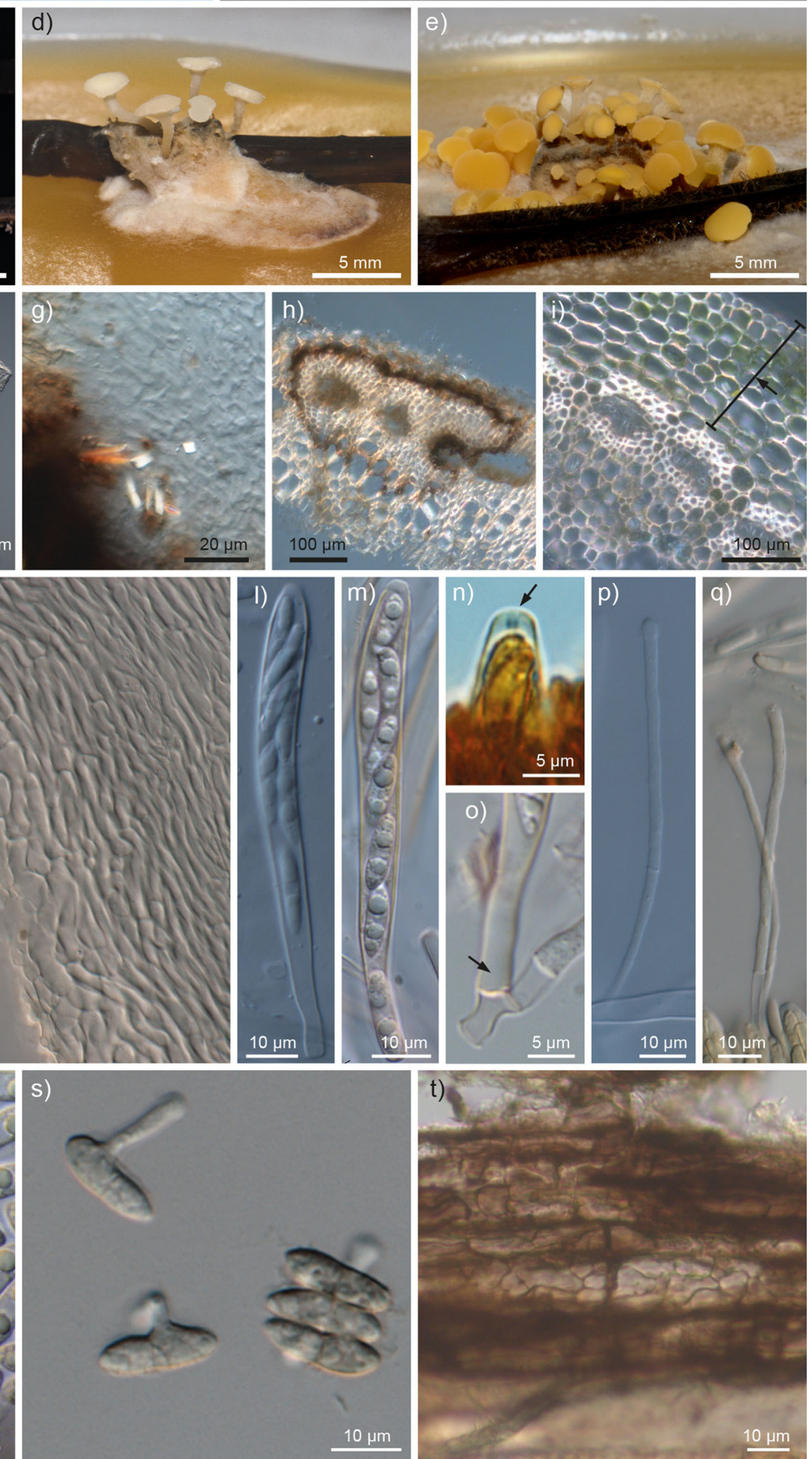


\section{Molecular characterization}

Phylogenetic analyses were based on four sequence markers (see below) and 12 different Hymenoscyphus species (including the new species)(Table 1). Herbarium specimens were provided by H.-O. Baral (private herbarium) and are available upon request. Hymenoscyphus menthae was chosen as an outgroup because of its bipolar symmetrical ascospores, which we consider to be more ancestral, and which differ from the rest of the included species that have distinctly heteropolar ("scutuloid") spores. Moreover, H. menthae shows a significantly high genetical distance from the rest of the included species.

DNA from lyophilized mycelium scraped off agar plates and from dried herbarium specimens (one ascocarp per specimen) was extracted with the NucleoSpin 96 plant II kit (Macherey-Nagel, Düren, Germany) using the CTAB-based lysis buffer PL1, in line with the manufacturer's protocol. The internal transcribed spacer (ITS), partial actin gene, partial elongation factor $1-\alpha$ gene (EF1- $\alpha$ ), and the partial calmodulin gene were amplified from the specimens and strains using the following reaction protocol: $10 \mu \mathrm{l}$ of total reaction volume, containing approximately $4 \mathrm{ng}$ template DNA, $2 \mu \mathrm{l} 5 \times$ reaction buffer, $0.2 \mathrm{mM}$ dNTPs, $0.5 \mu \mathrm{M}$ of forward and reverse primers, and 0.5 U GoTaq polymerase (Promega, Dübendorf, Switzerland). The PCR program started with an initial denaturation at $94{ }^{\circ} \mathrm{C}$ for $2 \mathrm{~min}$, followed by 35 cycles with $30 \mathrm{~s}$ denaturation at $94{ }^{\circ} \mathrm{C}, 30 \mathrm{~s}$ annealing at $50-60{ }^{\circ} \mathrm{C}$, and $60 \mathrm{~s}$ elongation at $72^{\circ} \mathrm{C}$, and ended with a final elongation of $5 \mathrm{~min}$ at $72^{\circ} \mathrm{C}$. PCR products were visualized on a $1.5 \%$ agarose gel and sequenced bi-directionally by a standard sanger-sequencing protocol. The primers used in this study were either newly designed or were published previously by other authors (White et al. 1990; Carbone and Kohn 1999; Grünig et al. 2007; Gross et al. 2014b). All primers are listed in Table 2, whereas Online Resource 1 provides the specific primer pair used for individual Hymenoscyphus species. Forward and reverse primer sequences were aligned using Geneious v6.1.3 (Biomatters Ltd, Auckland, New Zealand) and the primer sequences trimmed. All sequences were submitted to GenBank and the accession numbers can be drawn from Table 3 .

The ITS region of the co-occurring fungi of $H$. linearis on F. platypoda was amplified as described above, sequenced by Microsynth (Balgach, Switzerland), and characterized with NCBI-BLAST searches (http://blast.ncbi. nlm.nih.gov/Blast.cgi) (Table 4).

\section{Phylogenetic analysis}

A multiple sequence alignment of each individual marker was built using ClustalW, implemented in Geneious v6.1.3 with default parameters. The resulting alignments were checked by eye and minor corrections performed manually. Subsequently, ambiguously-aligned sites were removed systematically using Gblocks web v0.91b (http://molevol.cmima.csic.es/ castresana/Gblocks_server.html) through the application of less stringent options that allow for smaller final blocks, gaps in the final alignment and less strict flanking positions.

Bayesian inference (BI) and maximum likelihood (ML) phylogenetic analyses were performed with MrBayes v. 3.2.2 (Ronquist et al. 2012) and RAxML v.7.7.7 (Stamatakis 2006), respectively. Single sequence markers were subject to ML analysis to check for inter-marker conflicts in tree topologies. Subsequently, markers were concatenated for BI and ML analysis. Taxa with missing sequence data (e.g. H. albidoides) were also included in the dataset since both empirical and simulation studies have shown that phylogenetic inference of taxa with missing data is often estimated correctly (Wiens and Morrill 2011). Duplicate sequences were removed prior to phylogenetic analysis for both single and combined markers, however, these strains were manually added to tree figures post analysis. The PartitionFinder v1.1.0 (Lanfear et al. 2012) was used to determine the optimal substitution model and partitioning scheme of the concatenated dataset. This program uses predefined data blocks to find the best fit between the data and a particular substitution model and partitioning scheme. Therefore, we defined data blocks according to the intron-exon structure of the markers EF1- $\alpha$, calmodulin, and according to the intergenic spacer 1 and 2 and the 5.8S rDNA of the ITS marker. No blocks were defined for the actin gene fragment because the sequence is short and consists mainly of an intron. The branch length parameter was set to "linked" and the model was selected based on the Bayesian Information Criterion (BIC). The "greedy" search algorithm of PartitionFinder was used to determine the optimal partitioning scheme.

Analyses in MrBayes comprised of two parallel runs with four MCMC chains comprising 4,000,000 generations. The burn-in period was set to $25 \%$ of the total number of generations and trees were sampled every 500 generations. To ensure convergence of the two parallel runs we confirmed that the standard deviation of the split frequency was below 0.01 and examined the posterior probability plotted over the number of generations using the software tracer (Drummond and Rambaut 2008). ML analysis comprised of 500 initial independent ML inferences from the alignment file, while the tree with the best likelihood score was chosen to calculate support values based on 1,000 bootstrap replicates. Tree topologies were visualized using FigTree v1.4 (http://tree.bio.ed.ac.uk/software/figtree/) and rooted on H. menthae (H.B. 5846). Alignments and trees generated in this study were deposited in TreeBase (http://purl.org/phylo/treebase/phylows/study/ TB2:S16269). 
Table 1 List of samples that were incorporated in the phylogenetic analysis. NA indicates unavailable information

\begin{tabular}{|c|c|c|c|c|c|c|c|}
\hline Species & $\begin{array}{l}\text { Specimen/ Isolate } \\
\text { name }^{\mathrm{a}}\end{array}$ & Substrate & Country & $\begin{array}{l}\text { Latitude } \\
\left({ }^{\circ} \mathrm{N}\right)\end{array}$ & $\begin{array}{l}\text { Longitude } \\
\left({ }^{\circ} \mathrm{E}\right)\end{array}$ & $\begin{array}{l}\text { Collection } \\
\text { date/year }\end{array}$ & Collector \\
\hline H. albidoides & HMAS 264140 & $\begin{array}{l}\text { leaflets and leaflet } \\
\text { veins of } P \text {. quassioides }\end{array}$ & $\mathrm{CN}$ & NA & NA & 2011 and 2012 & H.D. Zheng \& W.Y. Zhuang \\
\hline H. albidoides & HMAS 264141 & $\begin{array}{l}\text { leaflets and leaflet } \\
\text { veins of } P \text {. quassioides }\end{array}$ & $\mathrm{CN}$ & NA & NA & 2012 and 2012 & H.D. Zheng \& W.Y. Zhuang \\
\hline H. albidoides & HMAS 264142 & $\begin{array}{l}\text { leaflets and leaflet } \\
\text { veins of } P \text {. quassioides }\end{array}$ & $\mathrm{CN}$ & NA & NA & 2013 and 2012 & H.D. Zheng \& W.Y. Zhuang \\
\hline H. albidus & Cas_01 & petioles of $F$. excelsior & $\mathrm{CH}$ & 46.47 & 8.94 & 2009 & V. Queloz \\
\hline H. albidus & Lav_01 & petioles of $F$. excelsior & $\mathrm{CH}$ & 46.44 & 8.84 & 2009 & V. Queloz \\
\hline H. albidus & Qui_01 & petioles of $F$. excelsior & $\mathrm{CH}$ & 46.5 & 8.72 & 2009 & V. Queloz \\
\hline H. caudatus & H.B. 7588c & cupules of Fagus sylvatica & $\mathrm{DE}$ & 48.540278 & 9.1205560 & $10 / 18 / 2004$ & H.O. Baral \& K. Siepe \\
\hline H. fraxineus & Aubo_03 & petioles of $F$. excelsior & $\mathrm{CH}$ & 46.51 & 6.37 & $4 / 3 / 2012$ & A. Gross \\
\hline H. fraxineus & Aubo_04 & petioles of $F$. excelsior & $\mathrm{CH}$ & 46.51 & 6.37 & $4 / 3 / 2012$ & A. Gross \\
\hline H. fraxineus & Aubo_06 & petioles of $F$. excelsior & $\mathrm{CH}$ & 46.51 & 6.37 & $4 / 3 / 2012$ & A. Gross \\
\hline H. fraxineus & CBS 122504 & petioles of $F$. excelsior & PL & 50.85 & 19.96 & 2000 & T. Kowalski \\
\hline H. fraxineus & Kore_06 & petioles of $F$. excelsior & HR & 44.75 & 15.7 & $8 / 29 / 2011$ & S. Stroheker \\
\hline H. fraxineus & Kore_07 & petioles of $F$. excelsior & HR & 44.75 & 15.7 & $8 / 29 / 2011$ & S. Stroheker \\
\hline H. fraxineus & Kore_08 & petioles of $F$. excelsior & HR & 44.75 & 15.7 & $8 / 29 / 2011$ & S. Stroheker \\
\hline H. fraxineus & Kore_09 & petioles of $F$. excelsior & HR & 44.75 & 15.7 & $8 / 29 / 2011$ & S. Stroheker \\
\hline H. fraxineus & Hanc_04 & petioles of $F$. excelsior & PL & 54.28 & 22.81 & 7/7/2012 & A. Gross \\
\hline H. fraxineus & Hanc_07 & petioles of $F$. excelsior & PL & 54.28 & 22.81 & $7 / 7 / 2012$ & A. Gross \\
\hline H. fraxineus & Hanc_08 & petioles of $F$. excelsior & PL & 54.28 & 22.81 & 7/7/2012 & A. Gross \\
\hline H. fraxineus & Hanc_09 & petioles of $F$. excelsior & PL & 54.28 & 22.81 & $7 / 7 / 2012$ & A. Gross \\
\hline H. fraxineus & Upps_02 & petioles of $F$. excelsior & SE & 59.85 & 17.65 & $10 / 10 / 2012$ & T. Kirisits \\
\hline H. fraxineus & Upps_03 & petioles of $F$. excelsior & SE & 59.85 & 17.65 & $10 / 10 / 2012$ & T. Kirisits \\
\hline H. fraxineus & Upps_05 & petioles of $F$. excelsior & SE & 59.85 & 17.65 & $10 / 10 / 2012$ & T. Kirisits \\
\hline H. fraxineus & Upps_09 & petioles of $F$. excelsior & SE & 59.85 & 17.65 & $10 / 10 / 2012$ & T. Kirisits \\
\hline H. fraxineus & Hokk_24 & petioles of $F$. mandshurica & JP & 42.67 & 141.6 & $5 / 13 / 2012$ & A. Gross/T. Hosoya \\
\hline H. fraxineus & Hokk_27 & petioles of $F$. mandshurica & JP & 42.67 & 141.6 & $5 / 13 / 2012$ & A. Gross/T. Hosoya \\
\hline H. fraxineus & Hokk_53 & petioles of $F$. mandshurica & JP & 42.61 & 141.45 & $5 / 13 / 2012$ & A. Gross/T. Hosoya \\
\hline H. fraxineus & Hokk_74 & petioles of $F$. mandshurica & JP & 42.95 & 141.15 & $5 / 14 / 2012$ & A. Gross/T. Hosoya \\
\hline H. fraxineus & Hokk_75 & petioles of $F$. mandshurica & JP & 42.95 & 141.15 & $5 / 14 / 2012$ & A. Gross/T. Hosoya \\
\hline H. fraxineus & Suga_27 & petioles of $F$. mandshurica & JP & 36.52 & 138.33 & $5 / 7 / 2012$ & A. Gross/T. Hosoya \\
\hline H. fraxineus & Suga_28 & petioles of $F$. mandshurica & JP & 36.52 & 138.33 & $5 / 7 / 2012$ & A. Gross/T. Hosoya \\
\hline H. fraxineus & Suga_44 & petioles of $F$. mandshurica & JP & 36.52 & 138.33 & $5 / 7 / 2012$ & A. Gross/T. Hosoya \\
\hline H. fraxineus & Suga_46 & petioles of $F$. mandshurica & JP & 36.52 & 138.33 & $5 / 7 / 2012$ & A. Gross/T. Hosoya \\
\hline H. fraxineus & Suga_50 & petioles of $F$. mandshurica & JP & 36.52 & 138.33 & $5 / 7 / 2012$ & A. Gross/T. Hosoya \\
\hline H. fructigenus & CBS 650.92 & cupules \& seeds of Quercus & $\mathrm{DE}$ & 47.619444 & 11.991667 & $9 / 6 / 1987$ & E. Weber \\
\hline H. fructigenus & H.B. 9594 & $\begin{array}{l}\text { cupule of Aesculus } \\
\text { hippocastanum }\end{array}$ & $\mathrm{DE}$ & 50.84 & 12.975 & $8 / 30 / 2011$ & B. Mühler \\
\hline H. linearis & Chic_01 & petioles of $F$. platypoda & JP & 35.94 & 138.77 & $5 / 22 / 2012$ & A. Gross \\
\hline H. linearis & Chic_02 & petioles of $F$. platypoda & JP & 35.94 & 138.77 & $5 / 22 / 2012$ & A. Gross \\
\hline H. linearis & Chic_03 & petioles of $F$. platypoda & JP & 35.94 & 138.77 & $5 / 22 / 2012$ & A. Gross \\
\hline H. linearis & Chic_04 & petioles of F. platypoda & JP & 35.94 & 138.77 & $5 / 22 / 2012$ & A. Gross \\
\hline H. linearis & Chic_05 & petioles of $F$. platypoda & JP & 35.94 & 138.77 & $5 / 22 / 2012$ & A. Gross \\
\hline H. linearis & Chic_06 & petioles of $F$. platypoda & JP & 35.94 & 138.77 & $5 / 22 / 2012$ & A. Gross \\
\hline H. linearis & Chic_08 & petioles of $F$. platypoda & JP & 35.94 & 138.77 & $5 / 22 / 2012$ & A. Gross \\
\hline H. linearis & Chic_09 & petioles of $F$. platypoda & JP & 35.94 & 138.77 & $5 / 22 / 2012$ & A. Gross \\
\hline H. linearis & Chic_11 & petioles of $F$. platypoda & JP & 35.94 & 138.77 & $5 / 22 / 2012$ & A. Gross \\
\hline H. linearis & Chic_12 & petioles of $F$. platypoda & JP & 35.94 & 138.77 & $5 / 22 / 2012$ & A. Gross \\
\hline H. linearis & Chic_13 & petioles of $F$. platypoda & JP & 35.94 & 138.77 & $5 / 22 / 2012$ & A. Gross \\
\hline
\end{tabular}


Table 1 (continued)

\begin{tabular}{|c|c|c|c|c|c|c|c|}
\hline Species & $\begin{array}{l}\text { Specimen/ Isolate } \\
\text { name }^{\mathrm{a}}\end{array}$ & Substrate & Country & $\begin{array}{l}\text { Latitude } \\
\left({ }^{\circ} \mathrm{N}\right)\end{array}$ & $\begin{array}{l}\text { Longitude } \\
\left({ }^{\circ} \mathrm{E}\right)\end{array}$ & $\begin{array}{l}\text { Collection } \\
\text { date/year }\end{array}$ & Collector \\
\hline H. linearis & Chic_15 & petioles of $F$. platypoda & $\mathrm{JP}$ & 35.94 & 138.77 & $5 / 22 / 2012$ & A. Gross \\
\hline H. linearis & Chic_16 & petioles of $F$. platypoda & JP & 35.94 & 138.77 & $5 / 22 / 2012$ & A. Gross \\
\hline H. linearis & Chic_17 & petioles of $F$. platypoda & JP & 35.94 & 138.77 & $5 / 22 / 2012$ & A. Gross \\
\hline H. linearis & Chic_18 & petioles of $F$. platypoda & JP & 35.94 & 138.77 & $5 / 22 / 2012$ & A. Gross \\
\hline H. menthae & H.B. 5846 & stem of Solidago canadensis & FL & 47.25 & 9.545833 & $7 / 8 / 1997$ & R. Wiederin et al. \\
\hline H. "phalaridis" & H.B. 8393 & culm of Poaceae & $\mathrm{DE}$ & 50.85 & 12.97780 & $12 / 19 / 2006$ & B. Mühler \\
\hline H. seminis-alni & H.B. 4974 & seeds of Alnus sp. & $\mathrm{DE}$ & 50.89 & 12.82 & $11 / 7 / 1993$ & M. Eckel \\
\hline H. serotinus & H.B. 8023 & twigs of Fagus sylvatica & ES & 41.219444 & -3.411111 & $11 / 21 / 2000$ & R. Galán et al. \\
\hline H. subferrugineus & H.B. 8013 & log of Populus x canadensis & $\mathrm{DE}$ & 51.591667 & 7.159722 & $12 / 13 / 2005$ & F. Kasparek \\
\hline H. subferrugineus & H.B. 8247 & twig of Corylus avellana & $\mathrm{CH}$ & 46.9850 & 8.284722 & $8 / 18 / 2006$ & E. Weber \\
\hline H. subferrugineus & H.B. 8392 & $\log$ of Populus alba & $\mathrm{DE}$ & 50.85 & 12.97780 & $12 / 19 / 2006$ & B. Mühler \\
\hline H. trichosporus & H.B. 6456 & branch of Alnus viridis & $\mathrm{DE}$ & 47.35278 & 10.22500 & $9 / 3 / 1999$ & L. Beenken \\
\hline
\end{tabular}

${ }^{\text {a }}$ Specimens in bold (all starting with H.B. available from H.-O. Baral), isolates in regular type

Inoculation studies on F. excelsior and F. platypoda

Fraxinus excelsior saplings were grown from the seeds of a single mother tree (from Zurich, Switzerland) and were 3 years old. Fraxinus platypoda saplings were 3-5 years old, 15-25 cm tall, and were collected from beneath different mother trees in the Chichibu forest (same site as $H$. linearis was collected). Both tree species were transferred into a climate chamber several months prior to inoculation under the following conditions: $10 \mathrm{~h}$ day at $18{ }^{\circ} \mathrm{C}, 45 \%$ relative humidity $(\mathrm{RH})$, and a light intensity of approx. $120-140 \mu \mathrm{Em}^{-2} \mathrm{~s}^{-1}$, $10 \mathrm{~h}$ night at $14{ }^{\circ} \mathrm{C}$ and $85 \% \mathrm{RH}$, with a $2 \mathrm{~h}$ transition at the start and end of the day, during which temperature, light, and RH increased and dropped gradually.
During the experiment, the climate chamber program was adjusted three times to simulate the winter resting phase or to induce the flushing of plants in spring, respectively: 7.11.2012, $7 \mathrm{~h}$ day at $10^{\circ} \mathrm{C}, 15 \mathrm{~h}$ night at $8{ }^{\circ} \mathrm{C}$ with a $1 \mathrm{~h}$ transition time (see above); $28.11 .2012,7 \mathrm{~h}$ day at $8^{\circ} \mathrm{C}, 15 \mathrm{~h}$ night at $6{ }^{\circ} \mathrm{C} .12 .03 .2013,10 \mathrm{~h}$ day at $15{ }^{\circ} \mathrm{C}, 10 \mathrm{~h}$ night at $12{ }^{\circ} \mathrm{C}$ with a $2 \mathrm{~h}$ transition time (see above). Two fungal strains (Chic_01, Chic_04) were grown on AMA medium supplied with pieces (approx. size: $1 \times 2 \times 5 \mathrm{~mm}$ ) of sterilized $\left(20 \mathrm{~min}\right.$ at $\left.120^{\circ} \mathrm{C}\right) \mathrm{F}$. excelsior sapwood. At the same time, similarly-sized sterile pieces of ash sapwood were placed on a sterile AMA plate for later use as negative control inocula. After approximately 2 months of incubation at $20^{\circ} \mathrm{C}$ in the dark, nine trees of each species were inoculated on 2 October 2012 as follows: using a sterile scalpel, a cut of about $2 \mathrm{~cm}$ in

Table 2 Primers used to amplify the four sequence markers used for phylogenetic analysis

\begin{tabular}{|c|c|c|c|}
\hline Name & Primer sequences $\left(5^{\prime}-3^{\prime}\right)$ & Target locus & Published by \\
\hline ITS1 & F: TCCGTAGGTGAACCTGCGG & Internal transcribed spacer $1 \& 2$ of the rDNA & White et al. 1990 \\
\hline ITS4 & R: TCCTCCGCTTATTGATATGC & Internal transcribed spacer $1 \& 2$ of the rDNA & White et al. 1990 \\
\hline CAL-228 F & F: GAGTTCAAGGAGGCCTTCTCCC & Calmodulin gene (partial) & Carbone and Kohn 1999 \\
\hline Hym_Cal_F & F: TYGATYCTTCAGCCTCATCT & Calmodulin gene (partial) & newly designed \\
\hline Hym_Cal_F2 & F: GGAYAAAGATGGTGACGGTTA & Calmodulin gene (partial) & newly designed \\
\hline CAL-737R & R: CATCTTTCTGGCCATCATGG & Calmodulin gene (partial) & Carbone and Kohn 1999 \\
\hline Hym_Cal_R & R: TGGTGCCATTGTTATCAGCGTCAACCT & Calmodulin gene (partial) & newly designed \\
\hline EF1-728 F & F: CATCGAGAAGTTCGAGAAGG & Elongation factor $1-\alpha$ gene (partial) & Carbone and Kohn 1999 \\
\hline EF1- $\alpha 1 \_R$ & R: GGGTTGTAGCCAACCTTCTTG & Elongation factor $1-\alpha$ gene (partial) & Grünig et al. 2007 \\
\hline EF1- $\alpha \_R 2$ & R: CAGCCTCGAACTCACCAGT & Elongation factor $1-\alpha$ gene (partial) & Grünig, unpublished \\
\hline Actin-512 F & F: ATGTGCAAGGCCGGTTTCGC & Actin gene (partial) & Carbone and Kohn 1999 \\
\hline Hp_actin_r & R: GGAGTCCTTTTGACCCATACC & Actin gene (partial) & Gross et al. 2014a, b \\
\hline
\end{tabular}

$F$ forward, $R$ reverse 
Table 3 GenBank accession numbers of all samples that were incorporated in the phylogenetic analyses. Accession numbers of previously unpublished sequences are in bold. NA specifies unavailable data

\begin{tabular}{|c|c|c|c|c|c|c|}
\hline Species & Specimen/ Isolate name & ITS & Calmodulin & $\mathrm{EF} 1-\alpha$ & Actin & Sequence origin \\
\hline H. albidoides & HMAS 264140 & KF188722 & KF188732 & NA & NA & Zheng et al. 2013 \\
\hline H. albidoides & HMAS 264141 & KF188721 & KF188731 & NA & NA & Zheng et al. 2013 \\
\hline H. albidoides & HMAS 264142 & KF188723 & KF188733 & NA & NA & Zheng et al. 2013 \\
\hline H. albidus & Cas_01 & GU586882 & GU586937 & GU586956 & KJ511015 & Queloz et al. 2011; Gross et al. 2014b \\
\hline H. albidus & Lav_01 & GU586884 & GU586938 & GU586957 & KJ511047 & Queloz et al. 2011; Gross et al. 2014b \\
\hline H. albidus & Qui_01 & GU586887 & GU586939 & GU586958 & KJ511048 & Queloz et al. 2011; Gross et al. 2014b \\
\hline H. caudatus & H.B. $7588 \mathrm{c}$ & KM114539 & KM114489 & KM114514 & KM114464 & this study \\
\hline H. fraxineus & Aubo_03 & KJ511173 & KJ511069 & KJ511121 & KJ511012 & Gross et al. 2014b \\
\hline H. fraxineus & Aubo_04 & KJ511174 & KJ511070 & KJ511122 & KJ511013 & Gross et al. 2014b \\
\hline H. fraxineus & Aubo_06 & KJ511175 & KJ511071 & KJ511123 & KJ511014 & Gross et al. 2014b \\
\hline H. fraxineus & CBS 122504 & FJ597975 & GU586951 & GU586970 & KJ511016 & Queloz et al. 2011; Gross et al. 2014b \\
\hline H. fraxineus & Kore_06 & KJ511201 & KJ511097 & KJ511149 & KJ511043 & Gross et al. $2014 b$ \\
\hline H. fraxineus & Kore_07 & KJ511202 & KJ511098 & KJ511150 & KJ511044 & Gross et al. $2014 \mathrm{~b}$ \\
\hline H. fraxineus & Kore_08 & KJ511203 & KJ511099 & KJ511151 & KJ511045 & Gross et al. $2014 \mathrm{~b}$ \\
\hline H. fraxineus & Kore_09 & KJ511204 & KJ511100 & KJ511152 & KJ511046 & Gross et al. $2014 b$ \\
\hline H. fraxineus & Hanc_04 & KJ511176 & KJ511072 & KJ511124 & KJ511018 & Gross et al. 2014b \\
\hline H. fraxineus & Hanc_07 & KJ511177 & KJ511073 & KJ511125 & KJ511019 & Gross et al. 2014b \\
\hline H. fraxineus & Hanc_08 & KJ511178 & KJ511074 & KJ511126 & KJ511020 & Gross et al. $2014 b$ \\
\hline H. fraxineus & Hanc_09 & KJ511179 & KJ511075 & KJ511127 & KJ511021 & Gross et al. 2014b \\
\hline H. fraxineus & Upps_02 & KJ511221 & KJ511117 & KJ511169 & KJ511065 & Gross et al. 2014b \\
\hline H. fraxineus & Upps_03 & KJ511222 & KJ511118 & KJ511170 & KJ511066 & Gross et al. $2014 \mathrm{~b}$ \\
\hline H. fraxineus & Upps_05 & KJ511223 & KJ511119 & KJ511171 & KJ511067 & Gross et al. 2014b \\
\hline H. fraxineus & Upps_09 & KJ511224 & KJ511120 & KJ511172 & KJ511068 & Gross et al. $2014 b$ \\
\hline H. fraxineus & Hokk_24 & KJ511194 & KJ511090 & KJ511142 & KJ511036 & Gross et al. 2014b \\
\hline H. fraxineus & Hokk_27 & KJ511195 & KJ511091 & KJ511143 & KJ511037 & Gross et al. $2014 b$ \\
\hline H. fraxineus & Hokk_53 & KJ511196 & KJ511092 & KJ511144 & KJ511038 & Gross et al. 2014b \\
\hline H. fraxineus & Hokk_74 & KJ511197 & KJ511093 & KJ511145 & KJ511039 & Gross et al. 2014b \\
\hline H. fraxineus & Hokk_75 & KJ511198 & KJ511094 & KJ511146 & KJ511040 & Gross et al. $2014 \mathrm{~b}$ \\
\hline H. fraxineus & Suga_27 & KJ511208 & KJ511104 & KJ511156 & KJ511052 & Gross et al. 2014b \\
\hline H. fraxineus & Suga_28 & KJ511209 & KJ511105 & KJ511157 & KJ511053 & Gross et al. $2014 b$ \\
\hline H. fraxineus & Suga_44 & KJ511215 & KJ511111 & KJ511163 & KJ511059 & Gross et al. 2014b \\
\hline H. fraxineus & Suga_46 & KJ511216 & KJ511112 & KJ511164 & KJ511060 & Gross et al. $2014 b$ \\
\hline H. fraxineus & Suga_50 & KJ511220 & KJ511116 & KJ511168 & KJ511064 & Gross et al. 2014b \\
\hline H. fructigenus & H.B. 9594 & KM114545 & KM114495 & KM114520 & KM114470 & this study \\
\hline H. fructigenus & CBS 650.92 & GU586933 & GU586952 & GU586971 & KJ511017 & Queloz et al. 2011; Gross et al. 2014b \\
\hline H. linearis & Chic_01 & KM114521 & KM114471 & KM114496 & KM114446 & this study \\
\hline H. linearis & Chic_02 & KM114522 & KM114472 & KM114497 & KM114447 & this study \\
\hline H. linearis & Chic_03 & KM114523 & KM114473 & KM114498 & KM114448 & this study \\
\hline H. linearis & Chic_04 & KM114524 & KM114474 & KM114499 & KM114449 & this study \\
\hline H. linearis & Chic_05 & KM114525 & KM114475 & KM114500 & KM114450 & this study \\
\hline H. linearis & Chic_06 & KM114526 & KM114476 & KM114501 & KM114451 & this study \\
\hline H. linearis & Chic_08 & KM114527 & KM114477 & KM114502 & KM114452 & this study \\
\hline H. linearis & Chic_09 & KM114528 & KM114478 & KM114503 & KM114453 & this study \\
\hline H. linearis & Chic_11 & KM114529 & KM114479 & KM114504 & KM114454 & this study \\
\hline H. linearis & Chic_12 & KM114530 & KM114480 & KM114505 & KM114455 & this study \\
\hline
\end{tabular}


Table 3 (continued)

\begin{tabular}{lllllll}
\hline Species & Specimen/ Isolate name & ITS & Calmodulin & EF1- $\alpha$ & Actin & Sequence origin \\
\hline H. linearis & Chic_13 & $\mathbf{K M 1 1 4 5 3 1}$ & $\mathbf{K M 1 1 4 4 8 1}$ & $\mathbf{K M 1 1 4 5 0 6}$ & $\mathbf{K M 1 1 4 4 5 6}$ & this study \\
H. linearis & Chic_15 & $\mathbf{K M 1 1 4 5 3 2}$ & $\mathbf{K M 1 1 4 4 8 2}$ & $\mathbf{K M 1 1 4 5 0 7}$ & $\mathbf{K M 1 1 4 4 5 7}$ & this study \\
H. linearis & Chic_16 & $\mathbf{K M 1 1 4 5 3 3}$ & $\mathbf{K M 1 1 4 4 8 3}$ & $\mathbf{K M 1 1 4 5 0 8}$ & $\mathbf{K M 1 1 4 4 5 8}$ & this study \\
H. linearis & Chic_17 & $\mathbf{K M 1 1 4 5 3 4}$ & $\mathbf{K M 1 1 4 4 8 4}$ & $\mathbf{K M 1 1 4 5 0 9}$ & $\mathbf{K M 1 1 4 4 5 9}$ & this study \\
H. linearis & Chic_18 & $\mathbf{K M 1 1 4 5 3 5}$ & $\mathbf{K M 1 1 4 4 8 5}$ & $\mathbf{K M 1 1 4 5 1 0}$ & $\mathbf{K M 1 1 4 4 6 0}$ & this study \\
H. menthae & H.B. 5846 & $\mathbf{K M 1 1 4 5 3 7}$ & $\mathbf{K M 1 1 4 4 8 7}$ & $\mathbf{K M 1 1 4 5 1 2}$ & $\mathbf{K M 1 1 4 4 6 2}$ & this study \\
H. "phalaridis" & H.B. 8393 & $\mathbf{K M 1 1 4 5 4 4}$ & $\mathbf{K M 1 1 4 4 9 4}$ & $\mathbf{K M 1 1 4 5 1 9}$ & $\mathbf{K M 1 1 4 4 6 9}$ & this study \\
H. seminis-alni & H.B. 4974 & $\mathbf{K M 1 1 4 5 3 6}$ & $\mathbf{K M 1 1 4 4 8 6}$ & $\mathbf{K M 1 1 4 5 1 1}$ & $\mathbf{K M 1 1 4 4 6 1}$ & this study \\
H. serotinus & H.B. 8023 & $\mathbf{K M 1 1 4 5 4 1}$ & $\mathbf{K M 1 1 4 4 9 1}$ & $\mathbf{K M 1 1 4 5 1 6}$ & $\mathbf{K M 1 1 4 4 6 6}$ & this study \\
H. subferrugineus & H.B. 8013 & $\mathbf{K M 1 1 4 5 4 0}$ & $\mathbf{K M 1 1 4 4 9 0}$ & $\mathbf{K M 1 1 4 5 1 5}$ & $\mathbf{K M 1 1 4 4 6 5}$ & this study \\
H. subferrugineus & H.B. 8247 & $\mathbf{K M 1 1 4 5 4 2}$ & $\mathbf{K M 1 1 4 4 9 2}$ & $\mathbf{K M 1 1 4 5 1 7}$ & $\mathbf{K M 1 1 4 4 6 7}$ & this study \\
H. subferrugineus & H.B. 8392 & $\mathbf{K M 1 1 4 5 4 3}$ & $\mathbf{K M 1 1 4 4 9 3}$ & $\mathbf{K M 1 1 4 5 1 8}$ & $\mathbf{K M 1 1 4 4 6 8}$ & this study \\
H. trichosporus & H.B. 6456 & $\mathbf{K M 1 1 4 5 3 8}$ & $\mathbf{K M 1 1 4 4 8 8}$ & $\mathbf{K M 1 1 4 5 1 3}$ & $\mathbf{K M 1 1 4 4 6 3}$ & this study \\
\hline
\end{tabular}

length was made in the bark and peripheral sapwood of stems in distal direction without removing the bark. Inocula were placed directly onto the sapwood under the incised bark and the wound covered with parafilm. Four trees were inoculated per fungal strain. In addition, one tree per species was inoculated with a sterile piece of sapwood, which served as negative control. Trees were checked weekly for the formation of necrotic lesions until the 7 May 2013 when tree health was assessed for the last time.

\section{Results}

Hymenoscyphus linearis Hosoya, A. Gross \& Baral, spec. nov. (Figs. 1, 2, and 3)

Mycobank: MB 810158
Etymology: referring to the linear pseudosclerotium from which apothecia emerge.

Teleomorph: (used signs: * living state; $\uparrow$, dead state)

Apothecia scattered, nail-shaped with a $0.7-3 \mathrm{~mm}$ long stipe (Fig. 1b-f), 1.5-5 mm in diam. (in nature), 1-3 mm (on AMA), arising from a black, linear pseudosclerotium (Fig. 1a-b), disc slightly concave to flat, whitish to pale cream in color (also bright yellow on AMA; Fig. 1e), becoming reddish when bruised in fresh state; pale orange $(155 \mathrm{PC}=\mathrm{C} 0 \mathrm{M} 12 \mathrm{Y} 32 \mathrm{~K} 0)$ with dark orange $(159 \mathrm{PC}=\mathrm{C} 1 \mathrm{M} 74 \mathrm{Y} 100 \mathrm{~K} 7)$ tint for the wounded part at the hymenium, more darkly-colored $(156 \mathrm{PC}=\mathrm{C} 0 \mathrm{M} 24 \mathrm{Y} 49 \mathrm{~K} 0)$ at the outside of the receptacle when dried, rehydrated pale orange all-over; stipe $0.3-3 \times 0.2-0.6 \mathrm{~mm}$ when dried; surface of stipe and receptacle smooth (but distinctly pubescent on AMA; Fig. 1e), whitish to pale cream, stipe base pale brown (dry dark brown) (Fig. 1c, f).

Table 4 ITS identification of fungi co-occurring on petioles of $F$. platypoda with $H$. linearis

\begin{tabular}{|c|c|c|c|c|c|}
\hline Strain name & $\begin{array}{l}\text { Sequence } \\
\text { length [bp] }\end{array}$ & Best NCBI-BLAST hit & Query coverage & Identities & Accession-Nr. \\
\hline Chic_101 & $393^{\mathrm{b}}$ & Marasmius capillaris (FJ596828.1) & $100 \%$ & $97 \%$ & KM516315 \\
\hline Chic_102 & 528 & $\begin{array}{l}\text { Colletotrichum acutatum (AJ749670.1)/Glomerella fioriniae } \\
\text { (JN121191.1) }\end{array}$ & $99 \% / 99 \%$ & $99 \% / 98 \%$ & KM516316 \\
\hline Chic_103 & 568 & Ceratobasidium sp. (DQ102442.1)/Rhizoctonia sp. (AY842391.1) & $100 \%$ & $99 \%$ & KM516317 \\
\hline Chic_104 & 511 & Nodulisporium sp. (KF881785.1) & $100 \%$ & $97 \%$ & KM516318 \\
\hline Chic_106-1 & 448 & Trichopeziza mollissima $(A B 481286)^{\mathrm{a}}$ & $100 \%$ & $99 \%$ & KM516319 \\
\hline Chic_106-2 & 487 & Fungal sp. (AB693768.1)/Phoma sp. ( HQ713778.1) & $100 \%$ & $99 \%$ & KM516320 \\
\hline Chic_107 & 568 & Ceratobasidium sp. (DQ102442.1)/Rhizoctonia sp. (AY842391.1) & $100 \%$ & $99 \%$ & KM516321 \\
\hline Chic_110-1 & $150^{\mathrm{b}}$ & Stemonitopsis subcaespitosa (JN123461.1) & $96 \%$ & $98 \%$ & NA \\
\hline
\end{tabular}

${ }^{a}$ A second hit (Trichopeziza sulphurea, JN033398) with $100 \%$ coverage and $99 \%$ identity proofed to be a false identification based on various other T. mollissima ITS comparisons

${ }^{\mathrm{b}}$ A large part of the ITS sequence not available due to sequencing problems 


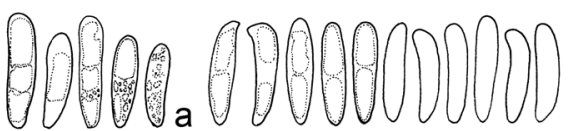

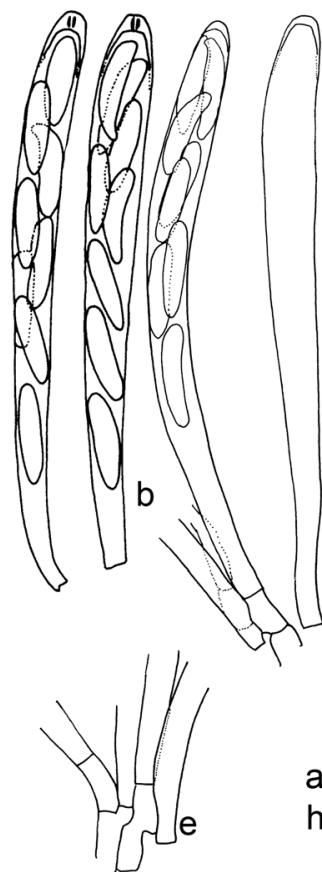
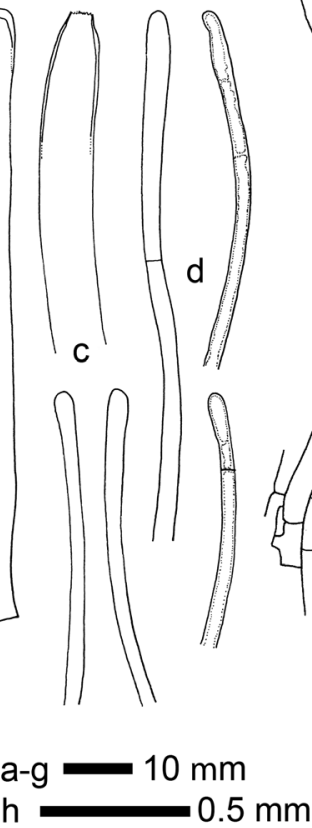

Fig. 2 Camera lucida illustration of Hymenoscyphus linearis (TNS-F48925, all in dead state): a Ascospores. First five from the left mounted in water, others mounted in $\mathrm{CB} / \mathrm{LA}$. Note that the variable pattern of spore contents is due to the dead state of the spores. b Asci. Two at the left mounted in MLZ. One at the right in CB/LA showing some ascogenous cells. c Ascus with dehiscent apex. d Paraphyses. Two at the right mounted in water, showing the internal structure of the dead state, while the left ones were mounted in lactic acid and only show the external

Pseudosclerotium forming a long and narrow straight, black line along the petiole (Fig. 1a-b), often starting from a leaflet scar (Fig. 1a), 0.5-0.8 mm wide in surface view, produced underneath the host epidermis (Fig. 1b, d), becoming apparent with removal of epidermis, in vertical section recognized as a black zone line of $10 \mu \mathrm{m}$ thickness (Fig. 1h), often formed around sclerenchyma cell layers,(Fig. 1h-i); rind in surface-view of textura epidermoidea (Fig. 1t).

Ectal excipulum of textura prismatica composed of rectangular cells, at lower flanks thick-walled (Fig. 2g), *15-21× 8-13(-15) $\mu \mathrm{m}, \dagger 15-20(-35) \times 5-10 \mu \mathrm{m}$ (Fig. 1k), cortical cells colored ochraceous (Fig. 1j), containing some large and small, globose, strongly refractive vacuolar bodies (VBs) in living state, contents turning orange-brownish in dead cells; cells at middle flanks becoming thinner-walled and smaller, toward the margin $\dagger 10-15 \times 5-8 \mu \mathrm{m}$, ending up in cylindrical cells at the margin (textura porrecta, Fig. $2 \mathrm{f}$ ); in section crystals observed among the cells at the base of the stipe (Fig. 1g).

Medullary excipulum of textura porrecta, composed of relatively thick-walled (up to $0.5 \mu \mathrm{m}$ ) hyphae of $\dagger 3-4 \mu \mathrm{m}$ in width, individual cells (15-)20-25(-30) $\mu \mathrm{m}$ long (Fig. 2g).

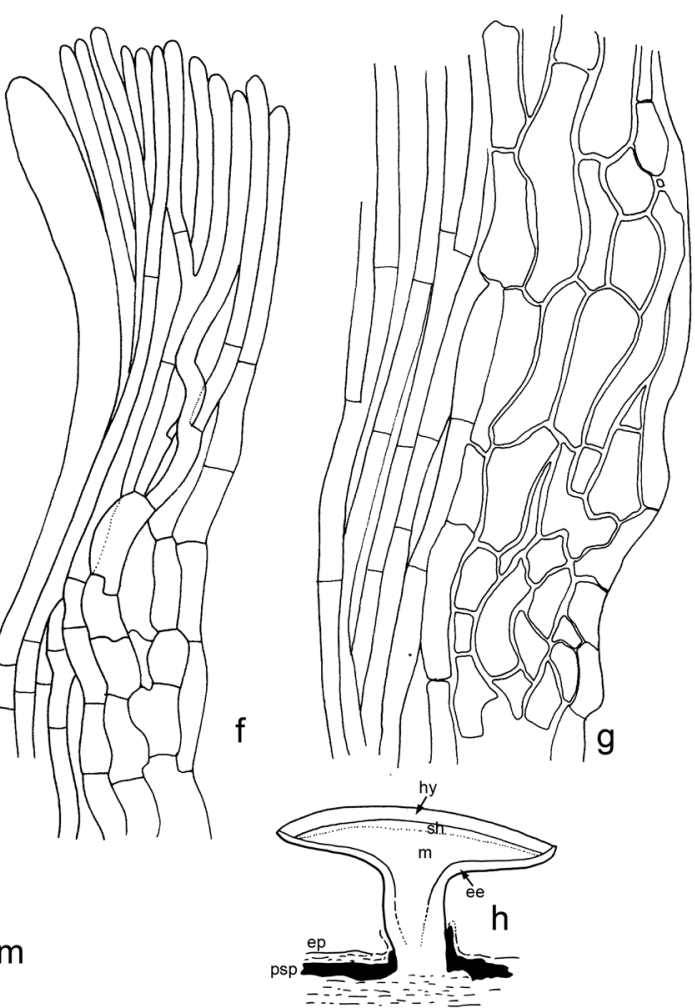

morphology. e Ascal bases showing the absence of croziers. f Vertical section through the margin showing the ectal excipular structure composed of thin-walled, prismatic, at upper margin hyphoid cells. $\mathbf{g}$ Ectal and medullary excipular structure from the middle flanks. $\mathbf{h}$ Median section of an apothecium showing different layers, basal stroma, and erumpent growth. hy hymenium, sh subhymenium, $m$ medullary excipulum, ee ectal excipulum, ep host epidermis, $p s p$ pseudosclerotial plate formed underneath the host epidermis

Asci $\uparrow 83-92 \times 7-8 \mu \mathrm{m}$, cylindric-clavate (Figs. $21-\mathrm{m}$ and $3 \mathrm{~b}$ ), 8-spored, arising from simple septa (Figs. 2o and 3e); apex subconical-subtruncate, thick-walled in dead state; with an amyloid ring forming two lines in lateral-view embedded in the lower part of the swollen ascus wall-thickening (Hymenoscyphus-type) (Figs. 2n and 3b), staining blue in MLZ or IKI without KOH-pretreatment.

Paraphyses cylindrical, straight, or slightly curved, sometimes expanding gradually toward the $\dagger 2-3 \mu \mathrm{m}$ wide apex, sometimes septate in the middle, densely septate below, multiguttulate in living specimen (strongly refractive vacuolar bodies, seen only in a few dead paraphyses when rehydrated), eguttulate in MLZ or CB/LA (Figs. 1q and 3d), contents turning orange-brownish in dead cells through oxidation.

Ascospores * $13.3-15 \times 4-4.4 \mu \mathrm{m}, \dagger 14-16 \times 3-4 \mu \mathrm{m}$ $(\dagger 14.8 \pm 0.7 \times 3.3 \pm 0.3$ in average $\pm \mathrm{SD}$, in $\mathrm{CB} / \mathrm{LA}$ mount), cylindrical to slightly ellipsoid, fusoid, or clavate, straight or flattened at one side, \pm beaked at the upper end when seen in profile-view, somewhat tapered at base, containing one large, globose oil drop 2.2-2.8 $\mu \mathrm{m}$ diam. in each half and mostly also one smaller drop $(1.3-1.8 \mu \mathrm{m})$ near each end, together 

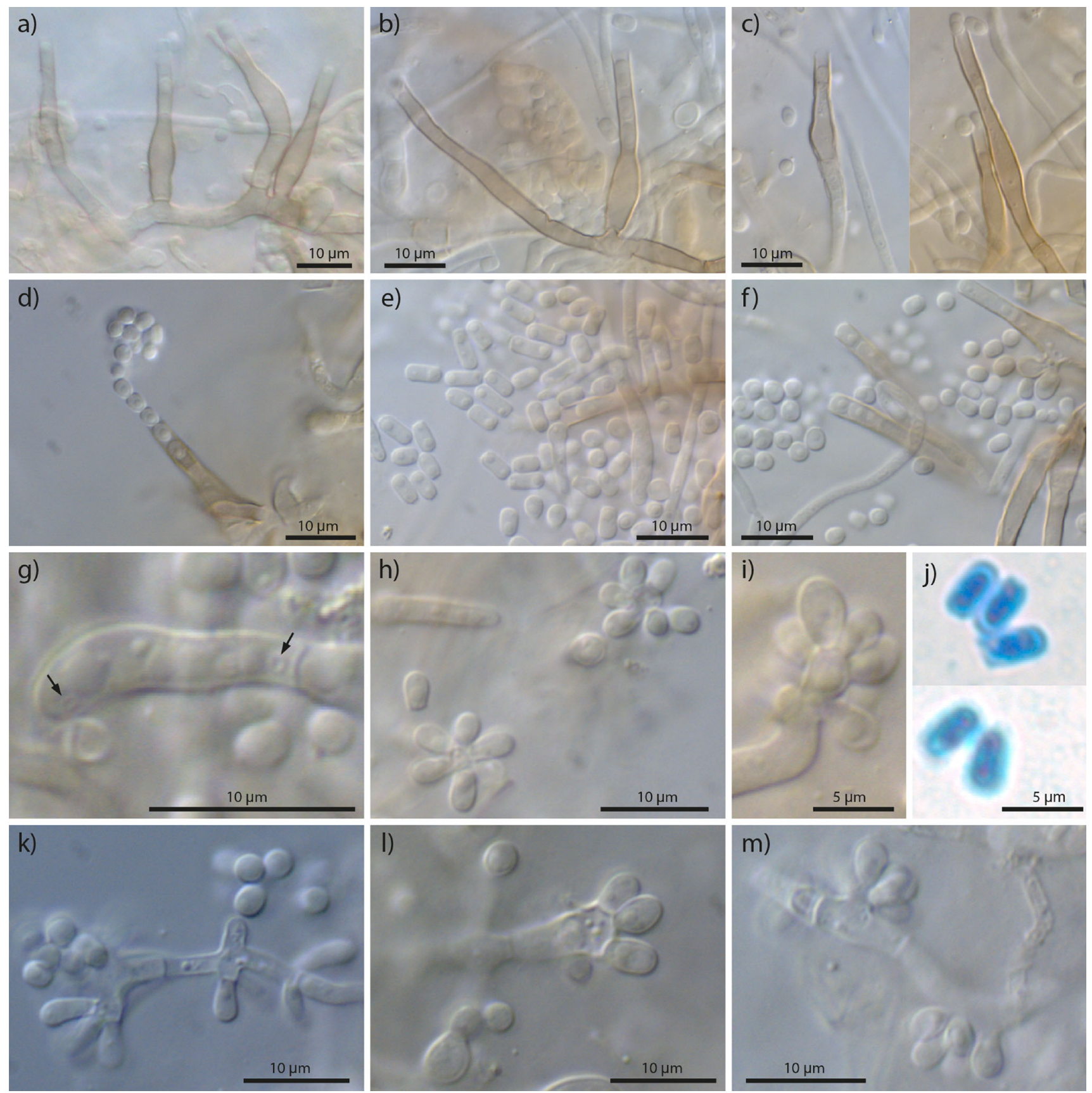

Fig. 3 Anamorph and synanamorph structures of $H$. linearis. a-c A selection of phialides showing variable shapes and sizes. d Phialide extruding a chain of conidia. e, f Conidia showing variable shapes and sizes. $\mathbf{g}$ Fertile cell showing a few subtle scars (arrows). h Top-view of a fertile cell only showing the laterally produced conidia which leads to a

flower-like appearance. i Side-view of a fertile cell showing multiple conidiogenous loci. j Conidia after 4 days of incubation on AMA, stained with lactophenol blue (dead state); no germination was observed. $\mathbf{k}-\mathbf{m}$ Variability of fertile cells

with a number of small droplets (when mounted in water, living state, Fig. 1r), oil drops often fused to form (1-)2 large elongate aggregations when observed in CB/LA (Fig. 2a); uninucleate (central globose transparent region in living state, Fig. 1r); surrounded by a delicate sheath that slips off the spore after discharge; biseriate in the upper portion of the dead asci while uniseriate to the base (Fig. 2b; probably overall biseriate inside living asci); hyaline while in the asci, becoming brown and often one-septate before germination (Fig. 1s). In most cases, ascospores germinated by producing one lateral germ tube in the middle of one of the two cells (Fig. 1s). 
Anamorph: Phialides rarely observed on pseudosclerotial structures in old cultures of the original isolation plates (Chic_01 and Chic_08) or after approx. 2 weeks of incubation at $20^{\circ} \mathrm{C}$ on the piece of inoculum used to revitalize strains Chic_01, Chic_04 and Chic_08 (which were stored at $4{ }^{\circ} \mathrm{C}$ for several months) on MEA or AMA. Phialides 16.7-36.7 ( $\varnothing=$ 24.0) $\mu \mathrm{m}$ long with Chalara-like appearance, produced alone or clustered, arising from slightly thick-walled, pale brown hyphae (Fig. 3a-c); venter mostly ellipsoidal, rarely cylindrical, 8.5-21 $(\varnothing=13.1) \times 3.6-5.2(\varnothing=4.3) \mu \mathrm{m}$, septate or aseptate at the base (Fig. 3a); collarette elongated, cylindrical, 7.5-16.5 $(\varnothing=11.6) \times 2.5-3 \quad(\varnothing=2.6) \mu \mathrm{m}$. Phialoconidia extruded in slimy droplets or rarely in short chains (Fig. 3d), one-celled, 2.1-5.9 $(\varnothing=3.6) \times 1.8-2.8(\varnothing=2.3) \mu \mathrm{m}$, cylindrical, slightly clavate to almost globose, often truncated at the base (Fig. 3e,f), hyaline, thin-walled $0.25 \mu \mathrm{m}$ (Fig. 3j), bearing 1-2 oil drops, these 0.5-1.4 $(\varnothing=1.1) \mu \mathrm{m}$. First formed conidium $6.8 \times 1.6 \mu \mathrm{m}$, short clavate with a truncated base. No germination of the conidia observed after 7 days on MEA and AMA.

Synanamorph: Sporotrichum-like, observed together with the Chalara anamorph during revitalization of strains Chic_01, Chic_04 and Chic_08 (see above). Fertile cells irregularly-shaped, hyaline or slightly pigmented, 8-9.8 $(-12.7) \times 3.1-4.5 \mu \mathrm{m}$, rarely showing subtle circular scars (Fig. 3g). Conidia hardly distinct from phialoconidia but distally rounded, holoblastic (monoblastic), or solitary thallic, arising in sympodial succession either from an intercalary cell, lateral branches or end cell (Fig. 3i,k,1,m).

Holotype: TNS-F-52072 (apothecia produced in moist chamber, see above) - Japan, Honshu, Saitama Pref., $20 \mathrm{~km}$ WSW of Chichibu, Tokyo Forests, the University of Tokyo, $1135 \mathrm{~m}, 35^{\circ} 56^{\prime} 11.7^{\prime \prime} \mathrm{N}, 138^{\circ} 48^{\prime} 9.55^{\prime \prime} \mathrm{E}$, leaf petioles of F. platypoda in deciduous tree forest where F. platypoda and Pterocarya rhoifolia dominate, 21-V-2012, leg. A. Gross. Culture Chic_Fk1_ss03=8601_01 (single spore isolate), deposited as NBRC 110603 and CBS 138754. ISOTYPE: ZT Myc 57167

Paratypes TNS-F-48369 (apothecia collected from nature) - Japan, Honshu, Saitama Pref., $20 \mathrm{~km}$ WSW of Chichibu, Tokyo Forests, the University of Tokyo, $35^{\circ} 56^{\prime}$ $11.7^{\prime \prime} \mathrm{N}, 138^{\circ} 48^{\prime} 9.55^{\prime \prime} \mathrm{E}, 1135 \mathrm{~m}$, leaf petioles of $F$. platypoda in a deciduous tree forest where F. platypoda and P. rhoifolia dominate, 11-IX-2012, leg. T. Hosoya. Culture FC-5043 (single spore culture obtained from apothecium), deposited as NBRC 110922; TNS-F-48366 - Japan, Honshu, Saitama Pref., $19.5 \mathrm{~km}$ WSW of Chichibu, Tokyo Forests, the University of Tokyo, $35^{\circ} 56^{\prime} 12.57^{\prime \prime} \mathrm{N}, 138^{\circ} 48^{\prime} 58.29^{\prime \prime} \mathrm{E}$, $855 \mathrm{~m}$, leaf petioles of $F$. platypoda in deciduous tree forest along with a valley where $F$. platypoda and $P$. rhoifolia dominate, 11-IX-2012, leg. T. Hosoya. Culture FC-5042 (singlespore culture obtained from the apothecium), deposited as NBRC 110921.
Diagnosis: $H$. linearis differs from all other species known within the $H$. fraxineus group due to the formation of a very narrow, straight, linear pseudosclerotium and the production of a synanamorph in culture. It further differs from $H$. fraxineus in its smaller apothecia and ascospores, the absence of croziers at the ascus base and the production of the teleomorph in culture, and from H. albidus in its slightly smaller ascospores and the formation of an anamorph in culture.

Phylogenetic analysis

The sequence information is complete for all treated species, except for $H$. albidoides (from which only ITS and calmodulin were available, because it was not possible to access the holotype specimen deposited in China). In addition, H. caudatus (H.B. 7588) and H. seminis-alni (H.B. 4974) lacked a small fragment less than $120 \mathrm{bp}$ at the 5'-end of the calmodulin sequence.

The alignment lengths of the different markers were as follows (alignment after removing ambiguously-aligned sites with GBlocks in brackets): ITS 493 bp (483 bp); actin 262 bp (242 bp), EF1- $\alpha 564$ bp (546 bp); calmodulin 405 bp (375 bp). The combined alignment upon removal of ambiguously-aligned sites was 1,646 bp long. Bootstrap support values of single-gene trees for the species $H$. fraxineus, $H$. albidus, $H$. albidoides, and $H$. linearis (hereafter termed $H$. fraxineus group) were mostly high and tree topologies congruent. The only exceptions were presented in $H$. fraxineus strains Hokk 75 and Suga 46, which are positioned in the $H$. albidus clade of the EF1- $\alpha$ phylogeny. In addition, the position of $H$. albidoides in the ITS and calmodulin gene tree was incongruent. In the ITS tree $H$. albidoides represents a sister species of $H$. fraxineus, whereas in the calmodulin tree, the species represents a sister species of $H$. albidus (Online Resource 2). Bootstrap support values of the other Hymenoscyphus species were mostly low $(<60 \%)$ and tree topologies incongruent. However, H. seminis-alni was positioned basal to the $H$. fraxineus group in all but the ITS gene phylogenies (Online Resource 2). PartitionFinder revealed the best BIC score for a model with five partitions for the analysis in MrBayes $(\mathrm{HKY}+\mathrm{G}, \mathrm{K} 80+\mathrm{I}, \mathrm{HKY}+\mathrm{G}, \mathrm{JC}$, and $\mathrm{K} 80+\mathrm{I}$, respectively) and three partitions for RAxML $(3 \times G T R+G)$. Using the combined dataset, Bayesian inference (BI) and maximum likelyhood (ML) tree topologies were fully congruent and branch-support values mostly high (Fig. 4). However, some ML bootstrap support values were lower compared to BI posterior probabilities. Hymenoscyphus linearis was separated from the other species with maximum support values, forming a monophyletic clade with the $H$. fraxineus group. The next most common ancestor of this group is represented by H. seminis-alni. 


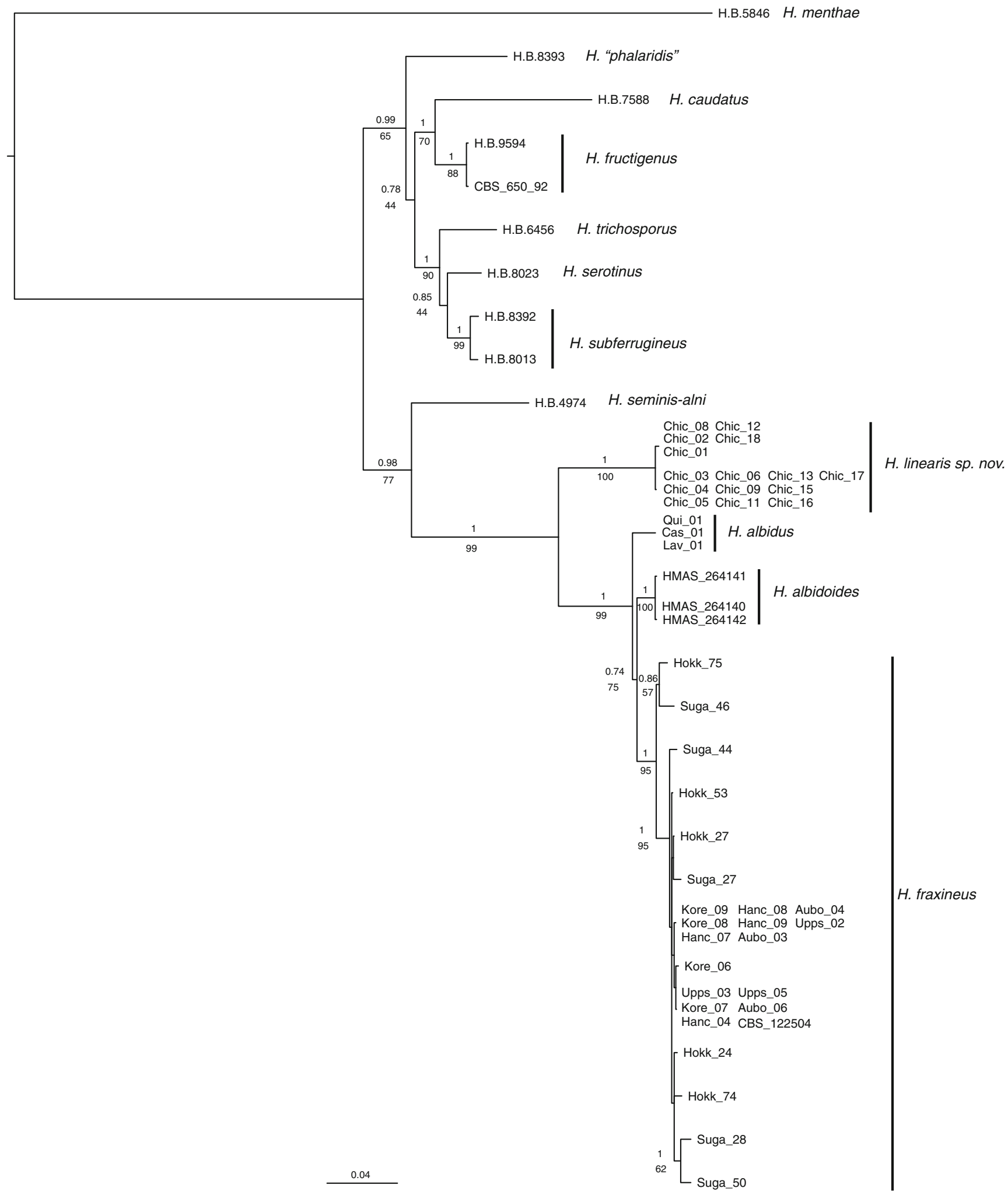

Fig. $4 \mathrm{BI}$ and ML phylogenetic analysis of the combined sequence alignments of the ITS rDNA, partial actin, EF1- $\alpha$, and calmodulin genes showing that H. linearis is separated from other Hymenoscyphus species and represents the closest relative of a lineage containing $H$. albidus, $H$. albidoides, and $H$. fraxineus. With the exception of $H$. seminis-alni, all other Hymenoscyphus species with scutuloid spores form a separate clade and are more distantly related. Bootstrap percentages of ML analysis are given below branches, while posterior probabilities of BI analysis are given above branches. The scale bar represents the number of substitutions per site 
Other fungi colonizing $F$. platypoda petioles

From 50 tissue samples next to linear pseudosclerotia on F. platypoda petioles, eight isolates were obtained and the ITS region sequenced. ITS sequencing revealed seven different taxa (Table 4).

Pathogenicity of $H$. linearis towards $F$. excelsior and F. platypoda

Five weeks after inoculation, callus formation was observed on all inoculated trees (including the negative controls) of both species. Approximately 5.5 months after inoculation, seedlings started to flush normally after the winter resting phase. Seven months after inoculation, the wounds at the inoculation sites were completely closed. No necrotic lesions were observed and the trees passed as completely healthy. One F. excelsior sapling died during the course of the experiment because of a latent infection with $H$. fraxineus, which had not been recognized at the time of inoculation.

\section{Discussion}

Phylogenetic analysis

The phylogenetic analysis presented in this paper identified Hymenoscyphus linearis as a member of a monophyletic group containing $H$. albidus, $H$. albidoides, and $H$. fraxineus (H. fraxineus group). Branch-lengths and branch-support values suggest that $H$. linearis is clearly differentiated from the other members of this group. In our study, the closest relative of the $H$. fraxineus group in the combined phylogeny is H. seminis-alni (see Baral 1996). It is the first time that this species was integrated in a phylogenetic analysis and no closer relative of the $H$. fraxineus group was identified in previous phylogenetic studies of this group (Zhao et al. 2012; Zheng and Zhuang 2013; Queloz et al. 2011). H. seminis-alni forms a black stroma in the innermost layer of the endosperm (Baral 1996) and closely resembles taxa of the $H$. fraxineus group. Besides what can be seen in molecular analyses, $H$. seminisalni differs from the others in its absence of crystals in the tissue of the stipe base and in the presence of conspicuous setulae on the ascospores. It should be noted that the ITS gene tree is in discordance with the combined tree. Inter-marker conflicts in single-gene phylogenies is a common phenomenon and can arise through different mechanisms, not necessarily questioning the species tree (Blair and Murphy 2011). The relationship between the other Hymenoscyphus species is not as well-established because branch support values for some nodes in the trees were low, and single-gene phylogenies were in partial disagreement. The quality of the multiplesequence alignment is crucial for inferring evolutionary history (Blair and Murphy 2011). However, finding markers with an optimal amount of sequence variation can be challenging (Rintoul et al. 2012). The sequence variation found across our sampled Hymenoscyphus taxa reached the upper-limit for constructing confident multiple-sequence alignments and this might have caused the abovementioned conflicts.

The genus Hymenoscyphus contains over 50 species when circumscribed under the exclusion of Cudoniella and Phaeohelotium (see Baral et al. 2013a), and is distributed worldwide (Kirk et al. 2008). It is characterized by medium-sized ascospores that are distinctly heteropolar in most of the species (basally-pointed, apically-rounded), with afairly pronounced bilateral symmetry (apically-beaked, referred to as "scutuloid" after one of its representatives, H. scutula), and also by its ability to form relatively long and narrow apothecial stipes. Further features, such as the characteristic shape of the amyloid ring of the ascus apex (Fig. 1n, Hymenoscyphus-type), the typically conspicuous refractive vacuolar bodies (VBs) in the paraphyses, and the prismatic texture of the ectal excipulum at the lower flanks are also found in closely related genera. The lack of a sufficient number of morphological distinguishing features, as well as a lack of good published keys, makes morphological identification difficult and often leads to false results. Molecular phylogenetic analysis helps in such cases, but several pitfalls complicate a molecular approach in most groups of ascomycetes, such as (i) the difficulty to isolate high quality DNA from herbarium specimens (Taylor and Swann 1994); (ii) a lack of appropriate (i.e. neutral, with enough variation) and readily available sequence markers (Rintoul et al. 2012); (iii) the frequency of misidentified herbarium material (Yang 2000; Plattner et al. 2009; Sokolski et al. 2004); (iv) inaccessible (as in the case of H. albidoides from China) or nonexistent (Plattner et al. 2009) type-specimens; and (v) the lack of GenBank reference sequences of many species. Consequently, only a few molecular studies of the genus Hymenoscyphus exist - most of them are based on ITS sequences and only examined a small subset of possible Hymenoscyphus species (Baral et al. 2006; Han and Shin 2008; Zhang and Zhuang 2004; Zhao et al. 2012; Queloz et al. 2011; Zheng and Zhuang 2013). This paper represents the first multi-gene phylogenetic study of 12 morphologically well-identified Hymenoscyphus species. The result suggests that the genus Hymenoscyphus is relatively diverse and possibly needs dividing into different genera or subgenera in the future. The current epidemic caused by $H$. fraxineus in Europe (Pautasso et al. 2013; McKinney et al. 2014; reviewed in: Gross et al. 2014a) calls for a better phylogenetic understanding of the genus Hymenoscyphus, but such a study requires the development of further molecular sequence markers of better quality. 


\section{Biology of $H$. linearis}

The biology of $H$. linearis appears similar to the other species within this group due to (i) occupation of a similar ecological niche on a closely related host, (ii) formation of a pseudosclerotium, and (iii) fructification in late summer. The observation that most of the linear pseudosclerotia are connected to a leaflet scar (Fig. 1a), suggests that the species infects leaflets, wherefrom it later invades the petioles. A pathogenic behavior is not known. Since conidia of anamorph and synanamorph states did not germinate, it is likely they act as spermatia, as proposed for conidia of $H$. fraxineus (Gross et al. 2012). The reported synanamorph is a novelty within the genus Hymenoscyphus and resembles Sporotrichum anamorphs of various Basidiomycetes (Carmichael 1980). Another similar and more closely related anamorph is known in connection with the fungus Xerombrophila crystallifera (Helotiaceae) (Baral et al. 2013b), but the two species differ slightly in conidia ontogeny. Xerombrophila crystallifera conidia have a more clearly blastic development, whereas $H$. linearis synanamorph conidia ontogeny lies midway between blastic and thallic making classification difficult. Preliminary matingtype analysis of $H$. linearis only identified the gene MAT1-2-1, while the other typical mating-type genes could not be found (A. Gross, unpublished). Furthermore, singlespore isolates readily formed apothecia in culture. Together, these suggest that $H$. linearis is a homothallic species capable of haploid selfing. However, unlike H. albidus, of which no anamorph state is known (Kirisits et al. 2013), H. linearis has the potential to outcross via spermatia. Homothallism in the H. fraxineus group may be related to the absence of croziers at the ascal base, because both $H$. albidus and $H$. linearis lack croziers.

Compared to $H$. albidus and $H$. fraxineus, $H$. linearis forms a very limited, narrow pseudosclerotium (Fig. 1a-b). One possible reason could be the existence of a strong fungal competitor that actively limits the growth of $H$. linearis. However, isolation of co-occurring fungi on $F$. platypoda petioles revealed seven different, inconsistently present taxa, without a dominant fungal competitor of $H$. linearis.

\section{A potential threat to European ash?}

The species of the $H$. fraxineus group (except $H$. albidoides) live on Fraxinus spp. and might have co-diverged with the Fraxinus species. However, as observed for $H$. fraxineus, they have the potential to occur on various Fraxinus spp. (Zhao et al. 2012; Drenkhan and Hanso 2010; Gross et al. 2014b). Hymenoscyphus albidoides is phylogenetically closely related to H. albidus and is the only species within this group that lives on a different host (Zheng and Zhuang 2013). This indicates that host-jumps, even to more distantly related tree species, have probably occurred in the past. Host-jumps following anthropogenic introduction are frequently linked to emerging diseases (Slippers et al. 2005). Likewise, emerging fungal diseases often result from host shift speciation (Giraud et al. 2010). Fungi's potential to form hybrids and the possible consequences thereof are evident (Olson and Stenlid 2002; Brasier 2000; Et-Touil et al. 1999; Stukenbrock 2013). Although $H$. linearis proved harmless in inoculation experiments on $F$. platypoda and $F$. excelsior, there remains a risk, albeit low, of hybridization with $H$. fraxineus upon introduction and this should therefore be avoided.

Acknowledgments We would like to thank N. Kamata for guidance in the Chichibu forest and W. Gams for his help regarding the description of the synanamorph. Further thanks go to O. Holdenrieder, T. Sieber and V. Queloz for interesting discussions and suggestions to M. Berchtold and S. Stroheker for excellent technical assistance and to C. Syrad for manuscript proofreading. We would also like to extend our thanks to the Genetic Diversity Center of ETH Zurich for providing laboratory facilities. This study was supported by a grant from the ETH Zurich (ETH-04 10-1).

Conflict of interest The authors declare that they have no conflict of interest.

\section{References}

Anonymous (2005) Pantone color bridge/coated. Pantone Inc., New Jersey

Baral HO (1996) Hymenoscyphus seminis-alni, a new species of the H. fructigenus-complex. Mycotaxon 60:249-256

Baral HO, Arenal F, Collado J, Galán R, López J, Peláez F, Platas G, Rubio V, Villarreal M (2006) Hynenoscyphus crataegi (Helotiales), a new species from Spain and its phylogenetic position within the genus Hymenoscyphus. Sydowia 58(2):145-162

Baral HO, Galán R, Platas G, Tena R (2013a) Phaeohelotium undulatum comb. nov. and Phaeoh. succineoguttulatum sp. nov., two segregates of the Discinella terrestris aggregate found under Eucalyptus in Spain: taxonomy, molecular biology, ecology and distribution. Mycosystema 32(3):386-428

Baral HO, Marson G, Bogale M, Untereiner WA (2013b) Xerombrophila crystallifera, a new genus and species in the Helotiales. Mycol Progr 12(3):475-488. doi:10.1007/s11557-012-0854-6

Baral HO, Queloz V, Hosoya T (2014) Hymenoscyphus fraxineus, the correct scientific name for the fungus causing ash dieback in europe. IMA Fungus 5(1):79-80. doi:10.5598/imafungus.2014.05.01.09

Blair C, Murphy RW (2011) Recent trends in molecular phylogenetic analysis: Where to next? J Hered 102(1):130-138. doi:10.1093/ jhered/esq092

Brasier CM (2000) Plant pathology: The rise of the hybrid fungi. Nature 405(6783):134-135

Brasier CM, Kirk SA (2010) Rapid emergence of hybrids between the two subspecies of Ophiostoma novo-ulmi with a high level of pathogenic fitness. Plant Pathol 59(1):186-199

Carbone I, Kohn LM (1999) A method for designing primer sets for speciation studies in filamentous ascomycetes. Mycologia 91(3): 553-556. doi:10.2307/3761358

Carmichael JW (1980) Genera of Hyphomycetes. Press, University of Alberta

Drenkhan R, Hanso M (2010) New host species for Chalara fraxinea. New Disease Reports 22:16 
Drummond A, Rambaut A (2008) Tracer [Online]. Available at: http:// beast.bio.ed.ac.uk/Tracer (accessed 25. October 2013).

Et-Touil A, Brasier CM, Bernier L (1999) Localization of a pathogenicity gene in Ophiostoma novo-ulmi and evidence that it may be introgressed from O. ulmi. Mol Plant-Microbe Interact 12(1):6-15. doi:10.1094/MPMI.1999.12.1.6

Giraud T, Gladieux P, Gavrilets S (2010) Linking emergence of fungal plant diseases and ecological speciation. Trends Ecol Evol 25(7): 387-395. doi:10.1016/j.tree.2010.03.006

Gross A, Holdenrieder O (2013) On the longevity of Hymenoscyphus pseudoalbidus in petioles of Fraxinus excelsior. Forest Pathol 43(2):168-170. doi:10.1111/efp.12022

Gross A, Zaffarano PL, Duo A, Grünig CR (2012) Reproductive mode and life cycle of the ash dieback pathogen Hymenoscyphus pseudoalbidus. Fungal Genet Biol 49(12):977-986. doi:10.1016/j. fgb.2012.08.008

Gross A, Holdenrieder O, Pautasso M, Queloz V, Sieber TN (2014a) Hymenoscyphus pseudoalbidus, the causal agent of ash dieback. Mol Plant Pathol 15(1):109-117

Gross A, Hosoya T, Queloz V (2014b) Population structure of the invasive forest pathogen Hymenoscyphus pseudoalbidus. Mol Ecol 23(12):2943-2960. doi:10.1111/mec. 12792

Grünig CR, Brunner PC, Duò A, Sieber TN (2007) Suitability of methods for species recognition in the Phialocephala fortinii-Acephala applanata species complex using DNA analysis. Fungal Genet Biol 44(8):773-788

Han J-G, Shin H-D (2008) Hymenoscyphus ginkgonis sp. nov. growing on leaves of Ginkgo biloba. Mycotaxon 103:189-195

Hosoya T, Otani Y, Furuya K (1993) Materials for the fungus flora of Japan (46). T Mycol Soc Jpn 34(4):429-432

Kirisits T, Dämpfle L, Kräutler K (2013) Hymenoscyphus albidus is not associated with an anamorphic stage and displays slower growth than Hymenoscyphus pseudoalbidus on agar media. Forest Pathol. doi:10.1111/efp.12042

Kirk PM, Cannon PF, Minter DW, Stalpers JA (2008) Ainsworth and Bisby's dictionary of the fungi. In, vol 10th edition. CAB International, Wallingford, $\mathrm{p} 771$

Kowalski T, Holdenrieder O (2009) The teleomorph of Chalara fraxinea, the causal agent of ash dieback. Forest Pathol 39(5):304-308

Lanfear R, Calcott B, Ho SYW, Guindon S (2012) PartitionFinder: Combined selection of partitioning schemes and substitution models for phylogenetic analyses. Mol Biol Evol 29(6):1695-1701. doi:10. 1093/molbev/mss020

Marčiulyniene D, Cleary MR, Shabunin D, Stenlid J, Vasaitis R Detection of Hymenoscyphus pseudoalbidus in Primorye region, far east Russia; COST Action FP1103 Fraxback 4th MC meeting \& workshop "Frontiers in ash dieback research", 4-6th of September 2013, Malmö, Sweden; accessed online 21.01.2014 http://www.fraxback.eu/. In, 2013. p 42

McKinney LV, Nielsen LR, Collinge DB, Thomsen IM, Hansen JK, Kjær ED (2014) The ash dieback crisis: genetic variation in resistance can prove a long term solution. Plant Pathol 63(3):485-499. doi:10. 1111 ppa. 12196

Olson Å, Stenlid J (2002) Pathogenic fungal species hybrids infecting plants. Microb Infect 4(13):1353-1359
Pautasso M, Aas G, Queloz V, Holdenrieder O (2013) European ash (Fraxinus excelsior) dieback - A conservation biology challenge. Biol Conserv 158:37-49. doi:10.1016/j.biocon.2012.08.026

Plattner A, Kim J-J, Reid J, Hausner G, Lim YW, Yamaoka Y, Breuil C (2009) Resolving taxonomic and phylogenetic incongruence within species Ceratocystiopsis minuta. Mycologia 101(6):878-887. doi: 10.3852/08-132

Queloz V, Grünig CR, Berndt R, Kowalski T, Sieber TN, Holdenrieder O (2011) Cryptic speciation in Hymenoscyphus albidus. Forest Pathol 41(2):133-142. doi:10.1111/j.1439-0329.2010.00645.x

Rintoul TL, Eggertson QA, Levesque CA (2012) Multigene phylogenetic analyses to delimit new species in fungal plant pathogens. In: Bolton MD, Thomma B (eds) Plant Fungal Pathogens: Methods and Protocols, vol 835. Methods in Molecular Biology. pp 549-569. doi:10.1007/978-1-61779-501-5 34

Ronquist F, Teslenko M, van der Mark P, Ayres DL, Darling A, Höhna S, Larget B, Liu L, Suchard MA, Huelsenbeck JP (2012) MrBayes 3.2: Efficient Bayesian phylogenetic inference and model choice across a large model space. Syst Biol 61(3):539-542. doi:10.1093/sysbio/sys029

Slippers B, Stenlid J, Wingfield MJ (2005) Emerging pathogens: fungal host jumps following anthropogenic introduction. Trends Ecol Evol 20(8):420-421. doi:10.1016/j.tree.2005.05.002

Sokolski S, Piché Y, Bérubé JA (2004) Lophodermium macci sp. nov., a new species on senesced foliage of five-needle pines. Mycologia 96(6):1261-1267

Stamatakis A (2006) RAxML-VI-HPC: maximum likelihood-based phylogenetic analyses with thousands of taxa and mixed models. Bioinformatics 22(21):2688-2690. doi:10.1093/bioinformatics/ bt1446

Stukenbrock EH (2013) Evolution, selection and isolation: a genomic view of speciation in fungal plant pathogens. New Phytol 199(4): 895-907. doi:10.1111/nph.12374

Taylor J, Swann E (1994) DNA from herbarium specimens. In: Hummel S (ed) Herrmann B. Ancient DNA, Springer New York, pp 166181. doi:10.1007/978-1-4612-4318-2_11

White TJ, Bruns T, Lee S, Taylor J (1990) Amplification and direct sequencing of fungal ribosomal RNA genes for phylogenetics. In: Innis MA et al (eds) PCR protocols: A guide to methods and applications. Academic Press, Inc., San Diego, USA and London, England, pp 315-322

Wiens JJ, Morrill MC (2011) Missing data in phylogenetic analysis: Reconciling results from simulations and empirical data. Syst Biol. doi:10.1093/sysbio/syr025

Yang ZL (2000) Revision of the Chinese Amanita collections deposited in BPI and CUP. Mycotaxon 75:117-130

Zhang Y-H, Zhuang W-Y (2004) Phylogenetic relationships of some members in the genus Hymenoscyphus (Ascomycetes, Helotiales). Nova Hedwigia 78(3-4):475-484. doi:10.1127/0029-5035/2004/ 0078-0475

Zhao Y-J, Hosoya T, Baral H-O, Hosaka K, Kakishima M (2012) Hymenoscyphus pseudoalbidus, the correct name for Lambertella albida reported from Japan. Mycotaxon 122:25-41. doi:10.5248/ 122.25

Zheng H-D, Zhuang W-Y (2013) Hymenoscyphus albidoides sp. nov. and H. pseudoalbidus from China. Mycol Progr 13(3):625-638. doi:10. 1007/s11557-013-0945-z 